\title{
Intraprocedural contrast-enhanced ultrasound (CEUS) in liver percutaneous radiofrequency ablation: clinical impact and health technology assessment
}

\author{
Giovanni Mauri • Emanuele Porazzi • Luca Cova • Umberto Restelli • Tania Tondolo • \\ Marzia Bonfanti • Anna Cerri • Tiziana Ierace • Davide Croce • Luigi Solbiati
}

Received: 30 July 2013 / Revised: 20 January 2014 / Accepted: 24 January 2014 /Published online: 22 February 2014

(C) The Author(s) 2014. This article is published with open access at Springerlink.com

\begin{abstract}
Objectives To assess the clinical and the economic impacts of intraprocedural use of contrast-enhanced ultrasound (CEUS) in patients undergoing percutaneous radiofrequency ablation for small $(<2.5 \mathrm{~cm})$ hepatocellular carcinomas.

Methods One hundred and forty-eight hepatocellular carcinomas in 93 patients were treated by percutaneous radiofrequency ablation and immediate assessment by intraprocedural CEUS. Clinical impact, cost effectiveness, and budget, organisational and equity impacts were evaluated and compared with standard treatment without intraprocedural CEUS using the health technology assessment approach.

Results Intraprocedural CEUS detected incomplete ablation in 34/93 $(36.5 \%)$ patients, who underwent additional treatment during the same session. At 24-h, complete ablation was found in 88/93 (94.6\%) patients. Thus, a second session of treatment was spared in 29/93 (31.1 \%) patients. Costeffectiveness analysis revealed an advantage for the use of intraprocedural CEUS in comparison with standard treatment
\end{abstract}

\section{G. Mauri $(\bowtie)$}

IRCCS Policlinico San Donato, Unit of Radiology, Piazza Malan 2-20097 San Donato Milanese, Milano, Italy

e-mail: vanni.mauri@gmail.com

E. Porazzi $\cdot$ U. Restelli $\cdot$ M. Bonfanti $\cdot$ D. Croce CREMS (Centre for Research on Health Economics, Social and Health Care Management), University Carlo Cattaneo-LIUC, Castellanza, VA, Italy

G. Mauri $\cdot$ L. Cova $\cdot$ T. Tondolo $\cdot$ A. Cerri $\cdot$ T. Ierace $\cdot$ L. Solbiati Azienda Ospedaliera Ospedale di Circolo di Busto Arsizio, Department of Oncology, Unit of Interventional Oncologic

Radiology, Busto Arsizio, Varese, Italy

U. Restelli $\cdot$ D. Croce

School of Public Health, Faculty of Health Sciences,

University of the Witwatersrand, Johannesburg, South Africa
$(4,639$ vs 6,592$)$ with a $21.9 \%$ reduction of the costs to treat the whole sample. Cost per patient for complete treatment was $€ 4,609$ versus $€ 5,872$ respectively. The introduction of intraprocedural CEUS resulted in a low organisational impact, and in a positive impact on equity

Conclusions Intraprocedural use of CEUS has a relevant clinical impact, reducing the number of re-treatments and the related costs per patient.

Teaching Points

- CEUS allows to immediately asses the result of ablation.

- Intraprocedural CEUS decreases the number of second ablative sessions.

- Intraprocedural CEUS may reduce cost per patient for complete treatment.

- Use of intraprocedural CEUS may reduce hospital budget.

- Its introduction has low organisational impact, and relevant impact on equity.

Keywords Radiofrequency ablation · Hepatocellular carcinoma $\cdot$ Cost-effectiveness $\cdot$ Clinical impact $\cdot$ Budget impact · Health technology assessment · Intraprocedural contrast-enhanced ultrasound

\section{Introduction}

Radiofrequency ablation (RFA) is widely accepted as a treatment for small hepatocellular carcinomas (HCC), with efficacy comparable to surgical resection for these lesions [1-6]. However, complete coagulation necrosis of the index tumour with a sufficient ablative margin is necessary before an RFA treatment can be considered complete $[7,8]$. If only partial necrosis is achieved, future re-treatment may be necessary, which may result in increased patient discomfort, greater 
technical difficulties, higher failure rates and an increased possibility of complications.

Lesion targeting and intraprocedural monitoring are generally performed using non-contrast-enhanced imaging, including ultrasound (US), computed tomography (CT) and magnetic resonance imaging (MRI). On the other hand, precise assessment of technical success requires the use of contrastenhanced imaging, and is generally performed using contrastenhanced CT (CE-CT) and/or contrast-enhanced MRI (CEMRI) within 1 week after the procedure [4, 9-12]. The use of contrast-enhanced US (CEUS) to assess the results of liver RFA has been described, and a diagnostic accuracy similar to CE-CT has been reported [13-18]. For this reason CEUS immediately after ablation has been proposed as an appropriate technique to identify possible incomplete ablation and the need for re-treatment within the same operative session [18, 19]. This approach could theoretically reduce the number of re-treatments required subsequently, with a potentially significant impact on both costs and patient outcomes.

At our institution, CEUS is always performed intraprocedurally immediately following ablation procedures and, when incomplete ablation is depicted, CEUS-guided targeted re-treatment is performed during the same treatment session. The aim of this study was to assess both the clinical and economic impact of intraprocedural CEUS in patients undergoing percutaneous RFA for HCC.

\section{Materials and methods}

\section{Patients}

A retrospective review was performed of consecutive patients that had undergone percutaneous thermal ablation of liver tumours at our institution between January 2008 and June 2011. Approval was obtained by the Institutional Review Board and patient consent was waived.

All patients with $\mathrm{HCC}$ lesions smaller than $2.5 \mathrm{~cm}$ in size treated by RFA were included in the analysis. At our institution, in all the patients with HCC smaller than $2.5 \mathrm{~cm}$ treatment is planned with a single insertion of the electrode. Patients were excluded if they had undergone treatment for metastatic tumours or had undergone prior treatment by microwave ablation. In addition, patients were excluded if their tumours were undetectable by US or if their tumours were larger than $2.5 \mathrm{~cm}$.

Procedure

Radiofrequency ablation with US guidance was performed under general anaesthesia, using a $3.5-\mathrm{MHz}$ probe with an incorporated guide and a 17-gauge cooled-tip electrode (CoolTip; Valleylab, Burlington, MA) with a 3-cm exposed portion.
All procedures were performed by two of three interventional radiologists with more than 10 years' experience in percutaneous thermal ablations and use of CEUS in the diagnostic and interventional field. CEUS was performed using $2.4 \mathrm{ml}$ sulphur hexafluoride microbubbles (SonoVue, Bracco, Italy) before and immediately after each RFA procedure to monitor and assess the therapeutic result before terminating the treatment session. For all examinations, contrast-specific software (Coherent Contrast Imaging $[\mathrm{CCI}]$ and Contrast Pulse Sequencing [CPS], Siemens Acuson, USA; ECI, Siemens, Germany; Contrast Tuned Imaging [CnTI], Esaote, Italy) in continuous mode with very low mechanical index (0.01-0.1) was employed.

Pre-treatment CEUS was performed as an initial step in the RFA session in order to reproduce lesion mapping on CE-CT and CE-MRI, and to allow real-time lesion targeting. Images and/or movie clips were digitally stored to be compared with the immediate post-ablation study. Immediate post-ablation evaluation using CEUS was performed 5-10 min after the assumed completion of the RFA session, with the patient still under general anaesthesia.

If residual enhancement was found in the ablated mass and/ or the volume of the devascularised area was considered too small to cover the entire tumour with a sufficiently thick safety margin, the treatment was considered incomplete and a new ablation was performed immediately during the same treatment session, as previously described [19]. CEUS was performed again after the new ablation to confirm the completeness of the treatment. CE-CT or CE-MRI was performed at $24 \mathrm{~h}$ in all cases and was used as the reference standard for assessment of technical success.

A case with positive findings of intraoperative CEUS that modified the treatment is shown in Fig. 1

\section{Clinical impact}

The number of patients in whom intraprocedural CEUS revealed incomplete treatment at the end of the ablation and who underwent a second CEUS-guided ablation in the same interventional session was retrospectively evaluated on the basis of the description of the procedure recorded in the operative registry. The number of incompletely treated lesions was determined by the 24-h post-ablation CE-CT or CE-MRI, read by the same three radiologists with more than ten years' experience. Patients in whom CEUS revealed incomplete ablation and who underwent a second CEUS-guided ablation in the same session with complete necrosis at 24-h follow-up were considered to have avoided a second intervention as a result of the intraprocedural use of CEUS.

Health technology assessment

The economic and managerial analysis was conducted using a health technology assessment approach, based on the 
Fig. 1 The case of a single HCC that had undergone RFA and immediate re-treatment because of the detection of residual unablated tumour with

intraprocedural CEUS. In the left hepatic lobe pre-treatment CE-CT (a) and CEUS (b) show an HCC with typical hypervascularity in arterial phase (arrowheads). c The HCC is treated with single insertion of RF electrode (arrowheads). d Gas produced by heating during ablation (arrowheads) seems to diffuse beyond the tumour margins. $\mathbf{e}$ Intraprocedural CEUS performed few minutes after electrode withdrawal demonstrates residual enhancing viable tumour (arrows) at the periphery of the volume of necrosis (arrowheads). f Second insertion of RF electrode is performed aiming at the area of residual enhancement (arrows) (dotted linepath of the electrode). g Post-ablation CEUS demonstrates large volume of necrosis (arrowheads) with complete ablation of the residual tumour previously detected (arrows). h Twenty-four-hour post-ablation CE-CT confirms that treatment is complete (arrowheadsablated zone)
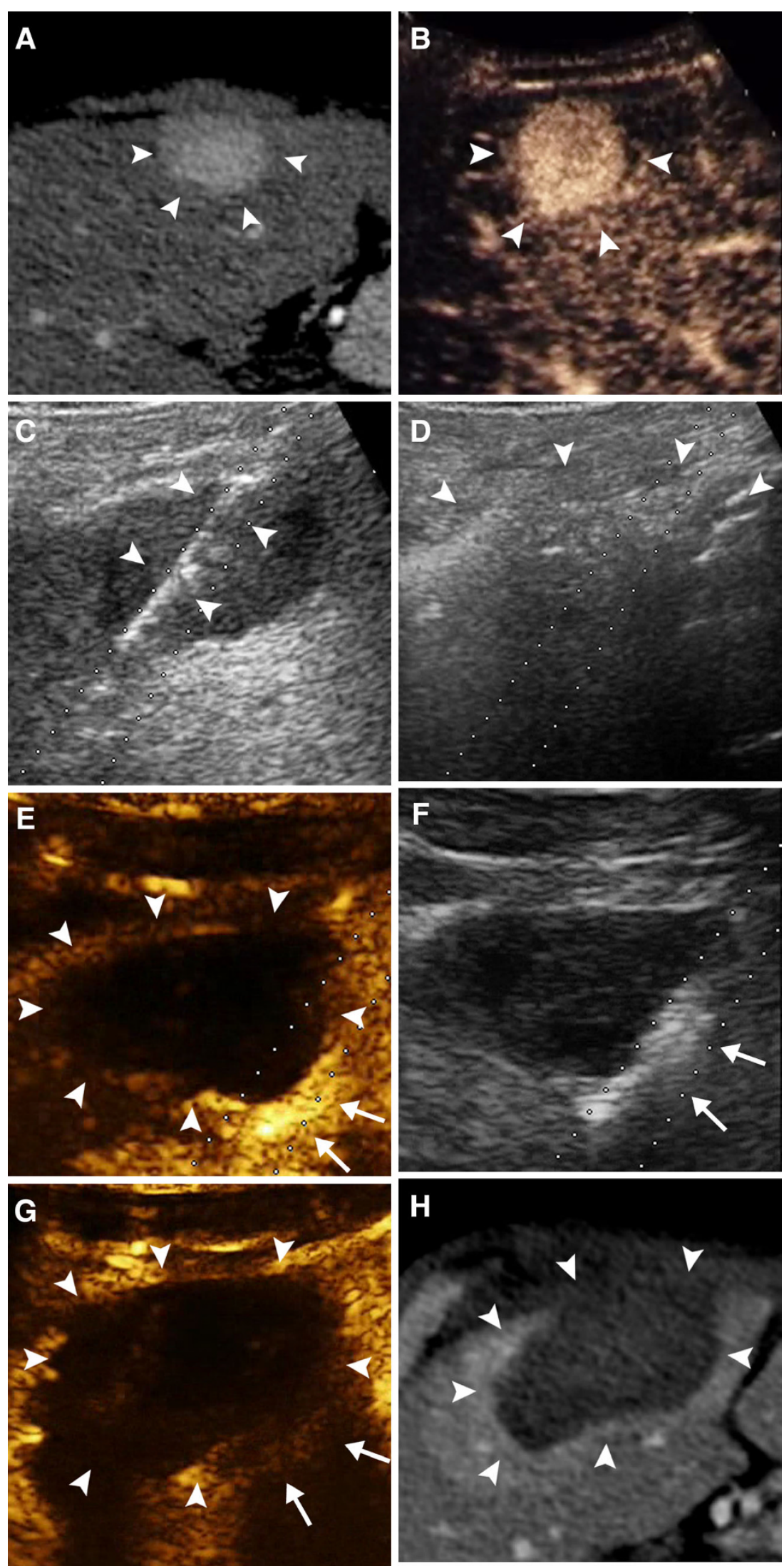
Guidelines for the Economic Evaluation of Health Technologies [20]. A cost-effectiveness analysis was conducted, along with a budget impact analysis, and analyses of the impacts on the organisation and on equity. The effectiveness parameter taken into account for the cost-effectiveness analysis was the percentage of patients who avoided a second session of treatment due to incomplete ablation.

The costs associated with the procedures were collected with a bottom-up approach, using Activity Based Costing [21-23]. Each procedure was divided into single phases and the related costs of each phase were evaluated, considering direct and indirect costs referring to human resources, machines (8-year amortisation), surgical instruments, consumption materials, drugs and overheads. All phases, from preadmission to patient discharge for a standard patient without complications, were evaluated using retrospective data related to the study sample in terms of time needed for percutaneous ablation, considering the number of lesions and length of hospitalisation. To assess procedure-related costs without intraprocedural CEUS, a differential analysis was performed, removing time and resources necessary to perform CEUS.

The perspective taken into consideration was that of the hospital. Cost data, provided by the management control service of the hospital, are value added tax inclusive and refer to 2011.

A sensitivity analysis was conducted to test the robustness of the results. One thousand simulations were performed, varying each cost category and effectiveness data with uniform distributions as follows: human resources $\pm 10 \%$; machinery $0,+750 \%$ (most of the technologies used for the intervention analysed completed the amortisation period, therefore a high range for sensitivity analysis was considered); laboratory exams $\pm 5 \%$; consumables $\pm 10 \%$; drugs $\pm 5 \%$; hotel services $\pm 5 \%$; overheads $0,-5 \%$; effectiveness $\pm 5 \%$. Cost variations were the same for the two procedures in each simulation, while effectiveness could have different values in the same simulation.

The impact on the hospital budget was calculated considering the annual number of RFA procedures for HCC performed in 2010. Two scenarios were than taken into consideration:

- Assessment of the impact of the use of CEUS, using the real number of interventions and reinterventions due to incomplete ablations within the period.

- Assessment of the impact of the procedure without intraprocedural CEUS, considering the treatments and the hypothetical number of re-treatments resulting from incomplete ablations.

The costs of each ablation and re-treatment were calculated for both procedures, along with the diagnosis-related group (DRG) reimbursement provided by the Regional Healthcare Service of Lombardy Region.
A sensitivity analysis was performed using bootstrapping methodology to simulate 100 different samples, considering a uniform distribution of $\pm 10 \%$ in the number of patients with one and two lesions.

The impact on the organisation was assessed through guided interviews with three experienced radiologists of the Interventional Oncologic Department of the hospital. It allowed quantification of the economic impact of the use of intraprocedural CEUS on the organisation and the perception of the short-term and medium/long-term organisational impact, using a seven-point scale (from highly negative to highly positive), compared with ablations without intraprocedural CEUS. The parameters taken into consideration were related to human resources, space needed, software or hardware needs, etc.

The impact on equity was investigated through guided interviews with the three experienced radiologists, detecting their perception using a seven-point scale concerning the two different processes in terms of adverse events, patient safety, accessibility, waiting lists, usability and invasiveness.

\section{Results}

During the course of the study, 384 patients underwent percutaneous RFA for 640 liver lesions. Of these, 148 HCC lesions in 93 patients met the inclusion criteria and entered our analysis. Among the 93 patients, 59 (63.4\%) were men and $34(36.6 \%)$ women, with an age range of $72.3 \pm 7.9$ years (median, 73.5 years). Treated HCC lesions had a maximum diameter of $1.86 \pm 0.57 \mathrm{~cm}$ (median, $2.0 \mathrm{~cm}$ ). A single HCC was treated in $58(62.4 \%)$ patients, while two, three and four HCC lesions were treated in $23(24.7 \%), 8(8.6 \%)$ and 4 (4.3\%) patients, respectively.

\section{Clinical impact}

Intraprocedural CEUS detected incomplete treatment after the first ablation in 43/148 (29.0 \%) lesions in 34/93 (36.5\%) patients. All 34 patients underwent additional treatment during the same session until complete absence of vascularisation was demonstrated by intraprocedural CEUS. At 24-h CE-CT or CE-MRI, complete ablation was found in 143/148 (96.6\%) lesions in 88/93 (94.6\%) patients. Only 5/93 (5.4\%) patients subsequently underwent local re-treatment. Thus, as a result of intraprocedural CEUS, a second session of treatment was spared in 29/93 (31.1\%) patients.

Health technology assessment

The results of the cost-effectiveness analysis are reported in Table 1. The mean cost of RFA without intraprocedural CEUS was $€ 4,228$, which was $3.7 \%$ less than RFA with 
Table 1 Cost-Effectiveness Analysis Results

\begin{tabular}{llllrr}
\hline Procedure & Mean cost $(€)$ & Effectiveness & $\Delta$ cost $(€)$ & $\Delta$ effectiveness & Cost effectiveness \\
\hline Without intraprocedural CEUS & 4,228 & $64.13 \%$ & 4,228 & $64.13 \%$ & 6,592 \\
With intraprocedural CEUS & 4,387 & $94.57 \%$ & +159 & $+30.43 \%$ & 4,639 \\
\hline
\end{tabular}

The mean cost per procedure is lower without the use of intraprocedural CEUS, while the effectiveness value has a substantial increase with the use of the aforementioned diagnostic procedure. The cost effectiveness value shows a lower, and then favourable, value for the procedure with the use of intraprocedural CEUS

intraprocedural CEUS. This difference was due to the longer time length of the RFA procedure and to the additional use of contrast agent.

Considering the effectiveness of RFA with and without intraprocedural CEUS in terms of avoided reinterventions, the use of intraprocedural CEUS led to a $94.57 \%$ value versus $64.13 \%$ (30.43 \% less) when CEUS was not employed. The cost-effectiveness assessment revealed a significant advantage to the use of intraprocedural CEUS $(4,639$ vs 6,592) (Fig. 2). Though more expensive, the procedure with intraprocedural CEUS proved to be more effective, with an incremental costeffectiveness ratio (ICER) (that represents the cost per effectiveness point gained) of 521.97 per intervention. The sensitivity analysis results referred to the ICER are reported in Fig. 3. The probability of intraprocedural CEUS being costeffective was over $50 \%$, with a willingness to pay a threshold of $€ 575$. This represents the willingness to pay of the Health Service to reach a 1-point effectiveness gain.

The budget impact analysis demonstrated that the use of intraprocedural CEUS allowed a reduction in terms of cost of

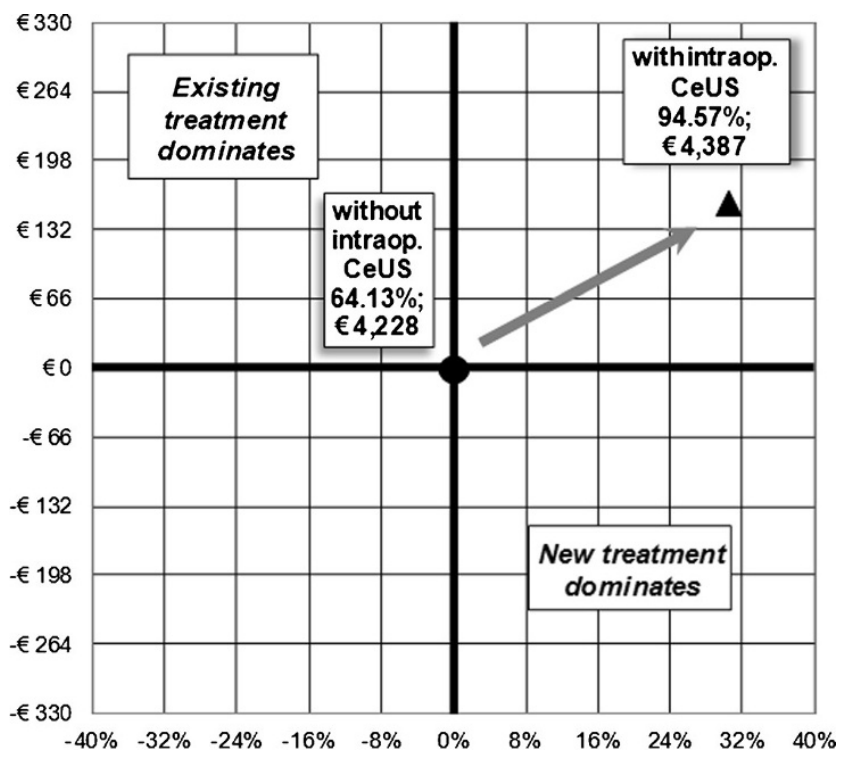

Fig. 2 Incremental cost-effectiveness plan. The plan shows the incremental cost and effectiveness of the procedure with intraoperational CEUS, compared with the standard procedure. There is an increase in effectiveness and in costs, the procedure being located in the North-East quadrant. The acceptability of the use of the procedure depends on the willingness to pay of the payer
$21.95 \%$ compared with treatment without intraprocedural CEUS, due to avoided re-treatments. A $25.95 \%$ reduction in terms of reimbursement was also observed, since the number of re-treatments in the sample considered is higher without intraprocedural CEUS and, therefore, the considered treatments and DRGs are higher in this scenario. The mean cost per patient for complete treatment (including first and second RFA) was $€ 4,609$ with the use of intraprocedural CEUS versus $€ 5,872$ for the standard procedure.

The sensitivity analysis revealed minimum and maximum cost reductions for the hospital of $19.02 \%$ and $23.26 \%$, respectively, with the use of intraprocedural CEUS.

The analysis of the organisational impact led to the identification of hypothetic investments necessary for the introduction of CEUS within an interventional radiology department of less than $€ 11,000$. Investments were considered to mainly involve software update, training of radiologists and meetings. The perceived short-term organisational impact results are reported in Fig. 4. In the short term, the introduction of CEUS leads to a medium negative impact on learning time, a low negative impact on training for personnel directly involved in the procedure, and for support personnel, meetings within the department (leading to an hypothetical loss of productivity for

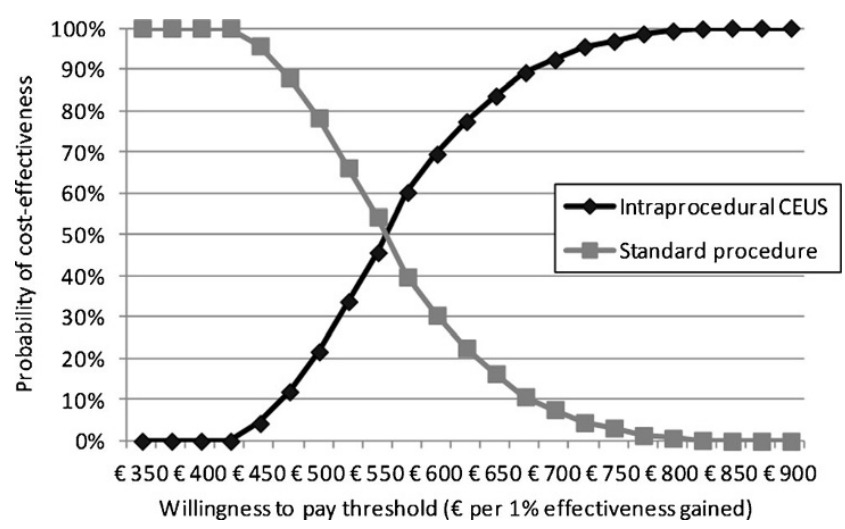

Fig. 3 Incremental cost-effectiveness ratio (ICER) sensitivity analysis results. The figure shows the percentage of the 1,000 ICERs calculated with the sensitivity analysis performed, which are cost effective (compared with the other procedure), considering hypothetical willingness to pay values for the regional healthcare service to increase the effectiveness of 1 unit. The cost effectiveness acceptability curve shows a probability higher than $50 \%$ for the procedure with intraprocedural CEUS to be costeffective, with a willingness to pay per additional effectiveness unit of $€ 575$ 
Fig. 4 Perceived short-term organisational impact. The use of intraprocedural CEUS leads to a short-term medium negative impact on learning time, and a low negative impact on training for personnel directly involved in the procedure, support personnel, meetings within the department and software update. It leads to a positive medium impact on the internal processes of the ward and appropriateness of requests for diagnostic exams, leading to a reduction in terms of further interventions or investigations needed for the same patient

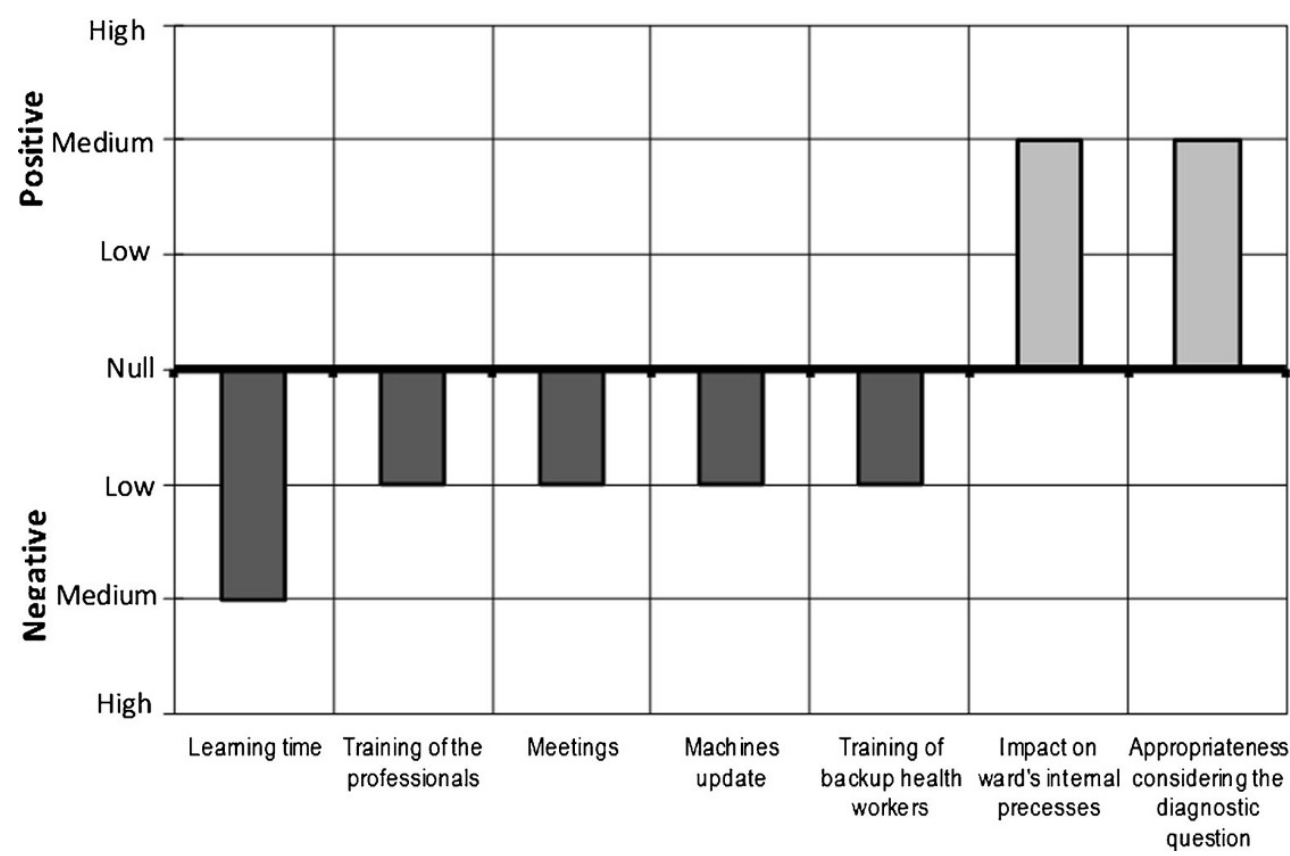

all the staff involved) and software update. A positive medium impact was determined for internal processes and appropriateness of requests for diagnostic exams, both leading to a reduction in terms of further interventions or investigations needed for the same patient. Considering a medium/long-term perspective, the positive impacts remain while the negative impacts do not further affect the organisation.

Considering the impact on equity, the use of intraprocedural CEUS was associated with a highly positive impact for the quality of data related to the investigated diagnostic question, which leads to more precise information to support medical decisions, a medium positive impact on reductions in waiting lists for surgical interventions, avoiding re-treatments, and a low negative impact on usability and invasiveness of the procedure.

\section{Discussion}

Our results show that the intraprocedural use of CEUS can reduce the number of incomplete treatments and consequently the number of re-treatments, as well as the total costs of RFA for HCC. RFA has been shown to be effective in the treatment of small HCC lesions, with results comparable to surgical resection [1-6]. However, given that the assessment of the completeness of ablation is generally performed after the treatment, often more than one ablation session is required to achieve complete response $[9,10]$, with increasing risk of complications for the patient and increasing costs. In our series we achieved complete ablation of $96.6 \%$ of lesions in $94.6 \%$ of patients at $24 \mathrm{~h}$, which is comparable with results reported in the literature [1-5].
CEUS can be performed in the operating room after the ablation, and has been used in different fields for the immediate assessment of completeness of ablation [19, 24]. Particularly, CEUS has been proven to be as accurate as other contrast-enhanced imaging modalities for the assessment of technical success after RFA [13-18]. For this reason some authors recommend CEUS immediately after RFA to permit re-treatment during the same interventional session if incomplete ablation is detected [17-19]. We adopted this strategy at our institution and, by using CEUS immediately after RFA, were able to detect incomplete ablation in $29.0 \%$ of lesions in $36.5 \%$ of patients. In these patients we were able to perform an immediate CEUS-guided targeted re-treatment that led to complete ablation in $94.6 \%$ of patients based on 24-h followup. Thus, a second session of treatment was avoided in $31.1 \%$ of patients. The need of a second intervention - that we performed in the same session - in our group is slightly higher than other series reported in the literature [1-9]. Several factors may have contributed to this result. Firstly, being a second-level centre, with high expertise in the treatment of liver diseases, patients are often referred to our department from other hospitals. Thus, our group included a high proportion of "complex cases" compared with cases at more general interventional radiology departments, with an increased mean technical difficulty of cases to be treated. Moreover, $37.6 \%$ of patients in our series were treated for more than one tumour in the same session. Third, the result of CEUS was judged by the interventional radiologist performing the treatment, and equivocal cases were generally considered as incompletely ablated, and underwent a second ablative treatment. Thus, the rate of incomplete treatment after the first ablation may have been slightly overestimated. Moreover, we finally 
achieved complete ablation at 24 hours in $94.6 \%$ of patients.

From an economic point of view, compared with the standard procedure, the introduction of intraprocedural CEUS resulted in a $3.7 \%$ increase in costs, due to a longer procedural time and the cost of the contrast agent. However, the use of intraprocedural CEUS resulted in better cost-effectiveness compared with the standard procedure and a higher probability of being cost-effective, with a $€ 575$ threshold of willingness to pay.

From a hospital perspective, the intraprocedural use of CEUS resulted in a $21.95 \%$ reduction in costs due to a lower number of re-treatments. This would result in the possibility of performing ablations on a greater number of patients, with consequently more effective use of resources and shortening of waiting lists. From the point of view of the Health Service, the use of intraprocedural CEUS allows a lower cost per patient for complete treatment, resulting in savings for the entire service.

The introduction of intraprocedural CEUS in centres where CEUS is already employed for other purposes would result in a low organisational impact, but to a relevant impact on equity, since the avoided re-treatments would have a positive impact on the quality-of-life of patients, decreasing their stress levels and reducing the number of hospitalisations.

Even though CEUS has a diagnostic accuracy similar to CE-CT in the assessment of treatment results [13-18], there are only few studies that compare CEUS with other contrastenhanced modalities immediately after RFA. A second possible limitation of our study is that being a second-level centre, with a larger number of "complex cases" compared with more general interventional radiology departments, the same analysis performed in a different hospital might lead to different results. Moreover, the decision to perform a new ablation immediately, in the same session, was made on the basis of the critical analysis of the result of intraprocedural CEUS made by the interventional radiologists. In some cases with an easy path to the target and a safely located tumour, even with equivocal result of incomplete ablation at CEUS a second ablation was performed. Thus, the need for immediate retreatment and, consequently, the clinical and economic impacts of CEUS, could have been overestimated in our series. As a consequence, unnecessary repetition of treatment in a significant number of patients would have happened. A fourth limitation is the absence of a true control group in which CEUS was not performed, as our results were obtained by comparing a real group in which CEUS was performed with a hypothetical one in which CEUS was not performed. A further bias in our study derives from the inclusion of only small $(<2.5 \mathrm{~cm}) \mathrm{HCCs}$ in our series.

In conclusion, the intraprocedural use of CEUS may have a relevant clinical impact in reducing the number of second ablative sessions needed to achieve a complete ablation of the tumour to be treated. Moreover, the intraprocedural use of CEUS may significantly reduce the costs per patient related to the percutaneous treatment with RFA of patients with HCC, being also characterised by a low organisational impact, and in a relevant impact on equity

Disclosure The authors declare no conflict of interest.

An unconditional grant was awarded by Bracco Imaging s.p.a., to cover the costs of the study.

Open Access This article is distributed under the terms of the Creative Commons Attribution License which permits any use, distribution, and reproduction in any medium, provided the original author(s) and the source are credited.

\section{References}

1. Hung HH, Chiou YY, Hsia CY, Su CW, Chou YH, Chiang JH et al (2011) Survival rates are comparable after radiofrequency ablation or surgery in patients with small hepatocellular carcinomas. Clin Gastroenterol Hepatol 9:79-86

2. Livraghi T (2010) Single HCC smaller than $2 \mathrm{~cm}$ : surgery or ablation: interventional oncologist's perspective. J Hepatobiliary Pancreat Sci $17: 425-429$

3. Cho YK, Rhim H, Noh S (2011) Radiofrequency ablation versus surgical resection as primary treatment of hepatocellular carcinoma meeting the Milan criteria: a systematic review. J Gastroenterol Hepatol 26:1354-1360

4. Livraghi T, Meloni F, Di Stasi M, Rolle E, Solbiati L, Tinelli C et al (2008) Sustained complete response and complications rates after radiofrequency ablation of very early hepatocellular carcinoma in cirrhosis: is resection still the treatment of choice? Hepatology 47: 82-89

5. Lencioni R, Cioni D, Crocetti L, Franchini C, Pina CD, Lera J et al (2005) Early-stage hepatocellular carcinoma in patients with cirrhosis: long-term results of percutaneous image-guided radiofrequency ablation. Radiology 234:961-967

6. Bruix J, Sherman M, Llovet JM, Beaugrand M, Lencioni R, Burroughs AK et al (2001) Clinical management of hepatocellular carcinoma. Conclusions of the Barcelona-2000 EASL conference. European Association for the Study of the Liver. J Hepatol 35:421-430

7. Kim YS, Lee WJ, Rhim H, Lim HK, Choi D, Lee JY (2010) The minimal ablative margin of radiofrequency ablation of hepatocellular carcinoma $(>2$ and $<5 \mathrm{~cm}$ ) needed to prevent local tumor progression: 3D quantitative assessment using CT image fusion. AJR Am J Roentgenol 195:758-765

8. Nakazawa T, Kokubu S, Shibuya A, Ono K, Watanabe M, Hidaka H et al (2007) Radiofrequency ablation of hepatocellular carcinoma: correlation between local tumor progression after ablation and ablative margin. AJR Am J Roentgenol 188:480-488

9. Terraz S, Cernicanu A, Lepetit-Coiffé M, Viallon M, Salomir R, Mentha $G$ et al (2010) Radiofrequency ablation of small liver malignancies under magnetic resonance guidance: progress in targeting and preliminary observations with temperature monitoring. Eur Radiol 20:886-897

10. Clasen S, Pereira PL (2008) Magnetic resonance guidance for radiofrequency ablation of liver tumors. J Magn Reson Imaging 27:421433

11. Kamei S, Matsuda J, Hagihara M, Kitagawa A, Izumi Y, Katsuda E et al (2012) Oblique approach for CT-guided liver radiofrequency 
ablation using multiplanar reformation images in hepatocellular carcinoma. Jpn J Radiol 30:533-539

12. Ishizaka H, Awata S, Arai H, Hirasawa S, Shimizu A (2010) CTguided radiofrequency liver tumour ablation: use of a two-step coaxial system with a fine guide needle wire unit for high-risk cases. Br J Radiol 83:1077-1079

13. Park MH, Rhim H, Kim YS, Choi D, Lim HK, Lee WJ (2008) Spectrum of CT findings after radiofrequency ablation of hepatic tumors. Radiographics 28:379-390

14. Vilana R, Bianchi L, Varela M, Nicolau C, Sánchez M, Ayuso C et al (2006) Is microbubble-enhanced ultrasonography sufficient for assessment of response to percutaneous treatment in patients with early hepatocellular carcinoma? Eur Radiol 16:2454-2462

15. Kisaka Y, Hirooka M, Koizumi Y, Abe M, Matsuura B, Hiasa Y et al (2010) Contrast-enhanced sonography with abdominal virtual sonography in monitoring radiofrequency ablation of hepatocellular carcinoma. J Clin Ultrasound 38:138-144

16. Lu MD, Yu XL, Li AH, Jiang TA, Chen MH, Zhao BZ et al (2007) Comparison of contrast enhanced ultrasound and contrast enhanced $\mathrm{CT}$ or MRI in monitoring percutaneous thermal ablation procedure in patients with hepatocellular carcinoma: a multi-center study in China. Ultrasound Med Biol 33:1736-1749

17. Gallotti A, D'Onofrio M, Ruzzenente A, Martone E, De Robertis R, Guglielmi A et al (2009) Contrast-enhanced ultrasonography
(CEUS) immediately after percutaneous ablation of hepatocellular carcinoma. Radiol Med 114:1094-1105

18. Meloni MF, Andreano A, Franza E, Passamonti M, Lazzaroni S (2012) Contrast enhanced ultrasound: should it play a role in immediate evaluation of liver tumors following thermal ablation? Eur J Radiol 81:e897-e902

19. Solbiati L, Ierace T, Tonolini M, Cova L (2004) Guidance and monitoring of radiofrequency liver tumor ablation with contrast-enhanced ultrasound. Eur J Radiol 51(Suppl):S19S23

20. Canadian Agency for Drugs and Technologies in Health (2006) Guidelines for the Economic Evaluation of the Health Technologies. CADTH, Ottawa

21. Cooper R, Kaplan RS (1988) Measure cost right: make the right decisions. Harv Bus Rev 66:96-103

22. Udpa S (1996) Activity Based Costing for Hospital. Health Care Manage Rev 21:83-96

23. Adam T, Evans DB, Murray CJL (2003) Econometric estimation of country-specific hospital costs. Cost Eff Resour Alloc $1: 3$

24. Mauri G, Cova L, Tondolo T, Ierace T, Baroli A, Di Mauro E et al (2013) Percutaneous laser ablation of metastatic lymph nodes in the neck from papillary thyroid carcinoma: preliminary results. J Clin Endocrinol Metab 98:E1203-E1207 